\title{
Plate distance effect on mixed convection in horizontal channels heated from below
}

\author{
G. Foglia, O. Manca \& S. Nardini \\ DIAM, Seconda Università degli Studi di Napoli, Aversa (CE), Italy
}

\begin{abstract}
Mixed convection in air in a horizontal channel with the lower wall heated at uniform heat flux is investigated experimentally. The study is accomplished for several heat fluxes, forced air velocities and two distances between the horizontal plates, $b=20 \mathrm{~mm}$ and $b=40 \mathrm{~mm}$. The Reynolds numbers are investigated between 5.0 and 250 , these being in the laminar regime. The Richardson number $\mathrm{Ri}=\mathrm{Gr} / \mathrm{Re}^{2}$ holds values in the range $3.0-7.4 \times 10^{4}$. The effect of the channel gap is investigated by flow visualization and wall temperature distribution.

Keywords: mixed convection, electronic cooling, thermal control, experimental investigation.
\end{abstract}

\section{Introduction}

Recently great attention has been focused on mixed convection in open-ended cavities for their wide use, such as thermal control of electronic equipments, chemical vapour deposition (CVD) of solid layer and solar collectors.

Buoyancy force due to the heating of the lower cavity wall induces secondary flows hence the local heat transfer increases. The onset point of the secondary flows is important because it delineates the region after which the twodimensional laminar flow becomes three-dimensional.

Understanding, manipulating and controlling the secondary motions in openended cavities are important. There is a need for further numerical and experimental investigations on three-dimensional mixed convection in cavities and particularly in horizontal channels. The relevant literature on mixed convection in horizontal channels heated from below has been recently reviewed in $[1,2]$. 
A bibliographical review on the mixed convection flows in horizontal rectangular ducts uniformly heated from below was presented by Nicolas [1]. A detailed synthesis of the recently discovered complex thermo-convective patterns was presented. An experimental study on flow patterns and heat transfer characteristics in a channel with heated bottom and the side walls was conducted by Zhang et al. [3]. It was to simulate finned heat sinks used in densely packed electronic enclosures. The bottom and side walls were heated and the top of the channel was cooled. The mixed convection of gas through a bottom heated horizontal plane channel was reviewed by Lin [2]. Results from theoretical, experimental and numerical explorations were examined in order to describe the various vortex flow patterns and the associated thermal characteristics in mixed convection at low Reynolds number. An investigation on the flow in mixed convection phenomenon of water in a horizontal rectangular duct, uniformly heated from below was accomplished experimentally and numerically by Bonnefoi et al. [4]. Many experiments were carried out for a wide range of fluid velocities and heat fluxes supplied to the wall in order to describe and to analyse the thermo-convective instabilities. An experimental investigation on mixed convection of air in a bottom heated horizontal flat duct by the top plate heating was carried out by Tseng et al. [5] to study the possible stabilization and elimination of the buoyancy driven unstable longitudinal, transverse and mixed vortex flow in mixed convection of air in a bottom heated horizontal flat duct by the top plate heating. An experimental investigation on mixed convection in air in inclined rectangular channels was presented by Ozsunar et al. [6]. Chen et al. [7] accomplished an investigation on mixed convection of air in a bottom heated horizontal flat duct by top plate heating experimentally, to study the possible stabilization and elimination of the buoyancy driven unstable longitudinal, transverse and mixed vortex flow.

Experiments for the onset and development of the buoyancy driven secondary air flow and enhancement of heat transfer in a horizontal convergent and a divergent channel were carried out by Liu and Gau [8]. A study on mixed convection in a horizontal channel with the lower wall heated at uniform heat flux was carried out by Manca et al. [9]. Flow visualization was performed to detect the flow patterns into the channel. Results showed that the separation from the lower heated plate strongly depended on the buoyancy force and forced velocity. Chen et al. [10] accomplished an experimental flow visualization combined with transient temperature measurement to explore the possible stabilization of the buoyancy drive vortex flow in mixed convection of air in a bottom heated horizontal flat duct by placing a rectangular solid block on the duct bottom.

Recently numerical and theoretical investigations were carried out on horizontal channels. Three-dimensional conjugate heat transfer in a rectangular duct with two discrete flush-mounted heat sources was studied numerically in the context of cooling of electronic equipments by Wang and Jaluria [11]. The magnitudes of the conduction and the convection transport were compared for different parametric combinations. Stability analysis for horizontal rectangular channels was carried out in $[12,13]$. A numerical study on mixed convection in a 
horizontal channel with the lower wall heated at uniform heat flux was carried out in transient regime, Buonomo et al. [14]. For high Reynolds numbers, if the upper channel wall was conductive, secondary motions were observed in terms of vortexes with longitudinal axes whereas conductive lower wall gave less marked secondary motions.

In this paper mixed convection in a horizontal channel, with the lower wall heated at uniform heat flux, is experimentally investigated. The upper parallel plate is unheated and made of glass. The present paper extends the investigation reported in [9] in order to examine the effect of the longitudinal channel aspect ratio. Flow visualization is performed to detect the flow patterns in the cavity. Wall temperature profiles along the axial coordinate are also presented.

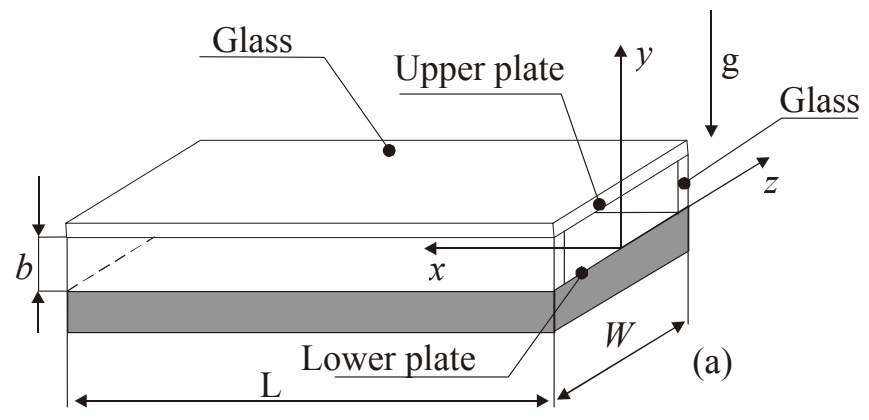

Figure 1: View of the heated part of the channel, the test section.

\section{Experimental apparatus}

The experimental test section was made up of a horizontal, uniformly heated wall and a parallel wall above, as reported in Fig.1. Each wall was made of two $400 \times 530 \mathrm{~mm}^{2}$ sandwiched phenolic fibreboard plates. The upper wall was a glass flat plate with a thickness of $3 \mathrm{~mm}$. The side walls of the channel were made of glass with a thickness of $3 \mathrm{~mm}$, which were machined to an accuracy of \pm 0.3 $\mathrm{mm}$, in order to take pictures of the flow motion. The heated channel was 400 $\mathrm{mm}$ long and $498 \mathrm{~mm}$ wide. The distance between the horizontal walls ranged from $20 \mathrm{~mm}$ to $40 \mathrm{~mm}$. The lower wall was made of two plates, the plate facing the channel was $3.2 \mathrm{~mm}$ thick and its surface adjacent to the internal air was coated with a $35 \mu \mathrm{m}$ thick nickel plated copper layer. The low emissivity of nickel (0.05) minimized radiation effects on heat transfer. The rear plate was 1.6 mm thick. Its back surface was coated with a $17.5 \mu \mathrm{m}$ thick copper layer, which also served as the heater. This is an electrical resistance obtained by cutting the copper layer in a serpentine shape. Its runs were $19.6 \mathrm{~mm}$ wide with a gap of nearly $0.5 \mathrm{~mm}$ between each one, giving the heater a total length of $9.0 \mathrm{~m}$. Its electrical current through the heater. In order to reduce conductive heat expected electrical resistance was $0.50 \Omega$. The lower wall was heated by passing a direct losses, a $150 \mathrm{~mm}$ Polystyrene block was affixed to the rear face of each principal 
wall. The narrow gaps between the runs, together with the relatively high thickness $(4.8 \mathrm{~mm})$ of the resulting low-conductive fibreglass were suitable to maintain a nearly uniform heat flux at the plate surface. Direct electrical current through the heaters was accomplished by using a Hewlett-Packard 6260B DC power supply. The supplied electrical power was evaluated by measuring the voltage drop across the plate and the current passing through it. An HP-3465A digital multi-meter was used to measure the voltage drop, whereas the current was calculated by the measured voltage drop across a reference resistance. The dissipated heat flux was evaluated to an accuracy of $\pm 2 \%$. The walls of the channel extensions were made of wood. A blower attached to the channel through a nozzle provided a variable mass flow rate. The entire apparatus was placed within a room in order to have a controlled ambient temperature. The experimental apparatus is schematically shown in Fig. 2.

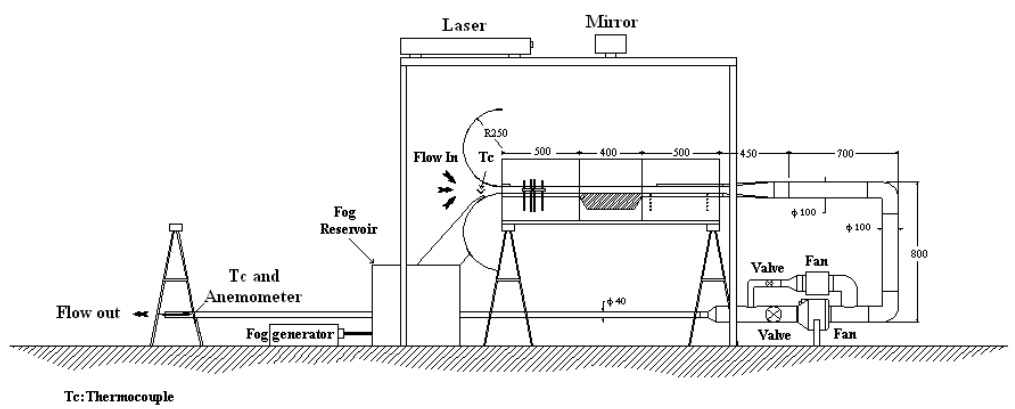

Figure 2: $\quad$ Layout of the experimental apparatus, dimensions in $\mathrm{mm}$.

Wall temperatures were measured by $0.50 \mathrm{~mm}$ OD ungrounded ironconstantan thermocouples embedded in each fibreboard plate and in contact with the outer layer. They were placed at twelve longitudinal stations at three different $\mathrm{z}$ values. A Kaye instrument K170 ice point was used as a reference for thermocouples junctions. Calibration of the temperature measuring system showed an estimated precision of the thermocouple-readout system of $\pm 0.1^{\circ} \mathrm{C}$.

Mass flow rate was calculated by measuring the velocity with a hot wire anemometer Dantec Mini CTA 54T30 with a 55P11 probe. The sensor was located at $2500 \mathrm{~mm}$ from the inlet section of a circular duct, with a diameter of $40 \mathrm{~mm}$, in order to have a fully developed laminar flow, Fig. 2. The range of velocity in this section, corresponding to the Reynolds number range between 5 and 1000 , varies between $0.042 \mathrm{~m} / \mathrm{s}$ and $8.6 \mathrm{~m} / \mathrm{s}$. The hot wire probe was calibrated at $15^{\circ} \mathrm{C}, 20^{\circ} \mathrm{C}$ and $25^{\circ} \mathrm{C}$ in the above velocity range. The maximum uncertainty in this range was about $4 \%$, the uncertainty on the measurement of the duct diameter was $1 \%$ and the uncertainty of the location of the sensor was $2 \%$. Smoke for visualization was generated by a fog oil generator. The smoke was passed into a plenum and its temperature was controlled with a thermocouple and its value was not more than $1{ }^{\circ} \mathrm{C}$ of the incoming air temperature, before entering into the channel. Then it was driven into the test 
section through a small slot situated along the lower edge of the inlet channel. The visualization was made possible by means of a laser sheet, generated by a He-Ne laser source. The laser sheet was produced by placing a mirror near the end of the test section at an angle of $45^{\circ}$ with respect to the direction of the main flow, after which a cylindrical lens was placed to enlarge the beam as desired. A still digital camera Nikon D-100 was used to take pictures.

\section{Data reduction}

The Grashof, Reynolds and Richardson numbers are defined as:

$$
\mathrm{Gr}=\frac{\mathrm{g} \beta \mathrm{q}_{\mathrm{c}} \mathrm{b}^{4}}{v^{2} \mathrm{k}}, \operatorname{Re}=\frac{\mathrm{u}_{\mathrm{i}} \mathrm{b}}{v}, \mathrm{Ri}=\frac{\mathrm{Gr}}{\mathrm{Re}^{2}}
$$

where $\mathrm{q}_{\mathrm{c}}$ is the average convective heat flux:

$$
\mathrm{q}_{\mathrm{c}}=\frac{1}{\mathrm{~L}} \int_{0}^{\mathrm{L}} \mathrm{q}_{\mathrm{c}}(\mathrm{x}) \mathrm{dx}
$$

the thermophysical properties were evaluated at the reference temperature

$$
\mathrm{T}_{\mathrm{r}}=\frac{\mathrm{T}_{\mathrm{w}}+\mathrm{T}_{0}}{2}
$$

with:

$$
\mathrm{T}_{\mathrm{w}}=\frac{1}{\mathrm{~L}} \int_{0}^{\mathrm{L}} \mathrm{T}_{\mathrm{w}}(\mathrm{x}) \mathrm{dx}
$$

where $\mathrm{T}_{\mathrm{w}}$ is the average wall temperature along the heated lower plate.

Local convective heat flux, $\mathrm{q}_{\mathrm{c}}(\mathrm{x})$, was not uniform because of radiation and conduction heat losses. Experimental data were reduced by first introducing, in the equations presented above, the local convective heat flux

$$
\mathrm{q}_{\mathrm{c}}(\mathrm{x})=\mathrm{q}_{\Omega}(\mathrm{x})-\mathrm{q}_{\mathrm{k}}(\mathrm{x})-\mathrm{q}_{\mathrm{r}}(\mathrm{x})
$$

where $\mathrm{q}_{\Omega}(\mathrm{x})$ is the local heat flux due to Ohmic dissipation, assumed uniform along $\mathrm{x}, \mathrm{q}_{\mathrm{k}}(\mathrm{x})$ is the local conduction heat losses from the plate and $\mathrm{q}_{\mathrm{r}}(\mathrm{x})$ is the local radiative heat flux from the plate. For each run, the terms $q_{k}(x)$ were calculated by means of a numerical procedure, a three-dimensional distribution of the temperature being assumed in the Polystyrene. Therefore, $\mathrm{q}_{\mathrm{k}}$ on the wall was a function of both $\mathrm{x}$ and $\mathrm{z}$ coordinates, and its values were averaged along $\mathrm{z}$. The predicted temperatures for some configurations of the system were previously compared with those measured by thermocouples embedded in the Polystyrene insulation and the relationship was very good, the maximum 
deviation being 3\%. A two-dimensional radiative cavity was made of the two plates, considered as diffuse-grey surfaces and two black edge sections at room temperature. In all cases, the radiative heat losses were not greater than $2 \%$ of the Ohmic dissipated power. The $\mathrm{q}_{\mathrm{r}}(\mathrm{x})$ terms were calculated for each temperature distribution of the wall, ambient temperature and plate spacing, by dividing each plate into sixteen strips along its length. Each strip was assumed at the spanwise average temperature.

The uncertainty in the calculated quantities was determined according to the standard single sample analysis recommended by Moffat [15]. The uncertainty of the Rayleigh, Reynolds and the average Nusselt numbers were $7 \%, 8 \%$ and $4 \%$ respectively.

\section{Results and discussion}

The experiments were carried out for $5.0 \leq \mathrm{Re} \leq 250$ and $1.86 \times 10^{4} \leq \mathrm{Ra} \leq 1.85 \times 10^{6}$ with corresponding Richardson number values from 3.00 to $7.4 \times 10^{4}$. The longitudinal aspect ratios were 10 and 20, whereas the transversal aspect ratio was 12.45 .

In Fig. 3, photos of flow visualization along a longitudinal section at $\mathrm{z}=0$, are shown. For $b=40 \mathrm{~mm}, \mathrm{Ra}=9.20 \times 10^{5}$ and $1.85 \times 10^{6}$ and for several $\mathrm{Re}$, it is observed that the increasing of forced motion, determines the movement of the flow separation point downstream, because inertial forces prevail on buoyancy forces for high Reynolds numbers. Whereas the separation point, $\mathrm{x}_{\mathrm{cr}}$ occurs as nearer the inlet section as higher the Ra value. For both Rayleigh number values, a backflow is observed, this is due to the low pressure area close to the upper wall. The fluid flow along the lower heated wall is laminar until it separates, after this point, the flow splits in two main currents; one going out the exit of the channel and another one comes back to the inlet section, both adjacent to the upper wall. At the lowest considered Reynolds number, downstream the flow separation point, the secondary motions are much more developed, whereas for highest Rayleigh numbers, Fig. 3b, the fluid flow seems chaotic.

In Fig. 4, for $b=20 \mathrm{~mm}$, and for the same heat flux of the previous case but for different Ra numbers, the separation point varies significantly and it is very close to the end of the heated channel, because inertial forces prevail on buoyancy forces as well as viscous forces prevail on inertial and buoyancy ones because $b$ is lower; so picture for $\mathrm{Re}=200$ is not shown because no flow separation is observed. As shown in Fig. 4b, the increase of heat flux determines the movement of the flow separation point upstream as expected. For low $\mathrm{Re}$ numbers, is still possible observed secondary motions while from $\mathrm{Re}=100$ to $\mathrm{Re}=250$ forced flow prevails.

In Fig. 5a, flow visualizations along a top view, at $\mathrm{y}=2 \mathrm{~mm}, 20 \mathrm{~mm}$ and 38 $\mathrm{mm}$, for $\mathrm{Ra}=9.20 \times 10^{5}, \mathrm{~b}=40 \mathrm{~mm}$ and $\mathrm{Re}=50$ are given. The fluid motion is almost laminar as shown at $\mathrm{y}=2 \mathrm{~mm}$ and according to the photos at $\mathrm{y}=20 \mathrm{~mm}$, the recirculation zone and unstable motion are noted. At $y=38 \mathrm{~mm}$, fluid flow is adjacent to the upper wall. For $\mathrm{Ra}=1.85 \times 10^{6}, \mathrm{Re}=50$ and at $\mathrm{y}=2 \mathrm{~mm}$, Fig. $5 \mathrm{~b}$, the fluid motion along the heated wall is laminar along axis up to about $100 \mathrm{~mm}$ 
where the separation starts. Smoke is not uniform due to velocity component along vertical direction that causes a cluster between streamlines. For $\mathrm{y}=20 \mathrm{~mm}$, the secondary motions are observed and photo at $\mathrm{y}=38 \mathrm{~mm}$ shows that, secondary motions are more irregular.

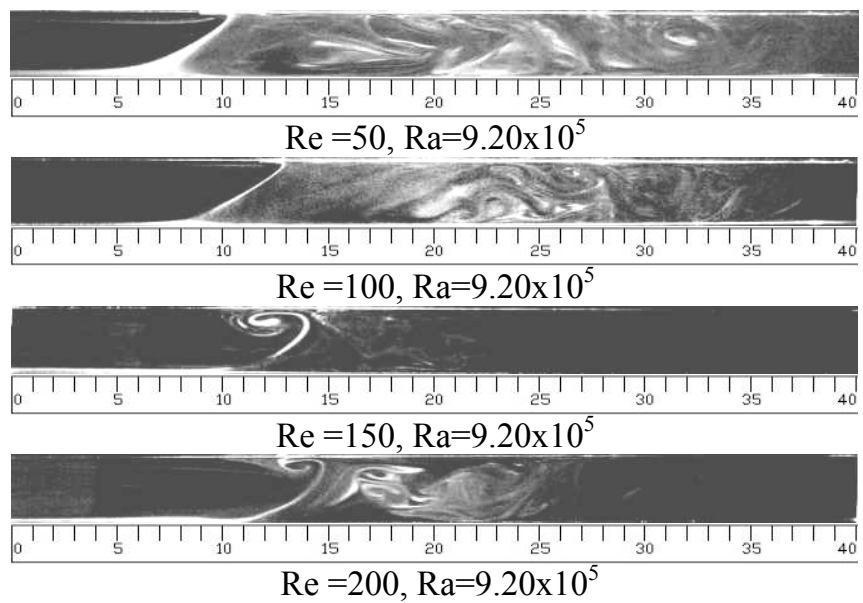

a)
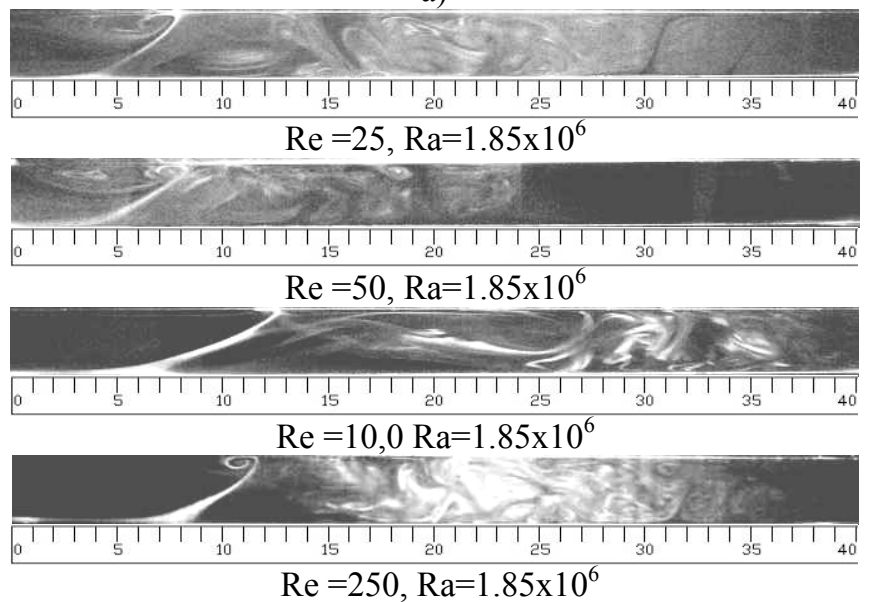

b)

Figure 3: Visualization of flow patterns at the longitudinal section $\mathrm{z}=0$ for $\mathrm{b}=40 \mathrm{~mm}$, different Reynolds numbers and: a) $\mathrm{Ra}=9.20 \times 10^{5}$, b) $\mathrm{Ra}=1.85 \times 10^{6}$.

The same top views photos are shown for $b=20 \mathrm{~mm}$, Fig. 6 . For $\mathrm{Ra}=1.86 \times 10^{4}$, Fig.6a, the flow is adjacent to the lower heated wall at $\mathrm{y}=2 \mathrm{~mm}$ and some effects of secondary motions are on the upper part of boundary layer, $y=10 \mathrm{~mm}$. The smoke close to the upper wall, $\mathrm{y}=18 \mathrm{~mm}$ indicates that secondary motions are developing and plumes are present along the transversal sections, furthermore 
some mushrooms-like structures begin to appear and than they becomes roller vortexes. For $\mathrm{Ra}=9.72 \times 10^{4}$, Fig. $6 \mathrm{~b}$, the trend is the same but secondary motion are clearly marked.

In Fig. 7, wall temperature rise to the ambient temperature on the heated wall are reported as a function of the $\mathrm{x}$ coordinate for two Rayleigh number values and $b=40 \mathrm{~mm}$. Wall temperature decreases for high Reynolds numbers as expected. Figure $7 \mathrm{~b}$ shows that wall temperature profiles, for high Rayleigh numbers, are very similar to the ones observed at low Rayleigh numbers up to $\mathrm{Re}=100$, whereas an almost uniform profile in the centre and a maximum temperature value close to $x=300 \mathrm{~mm}$ for $\mathrm{Re}=250$ is noticed. For $b=20 \mathrm{~mm}$, Fig. 8 , temperature are low due to the smaller channel gap that causes a better external heat transfer on the upper wall.

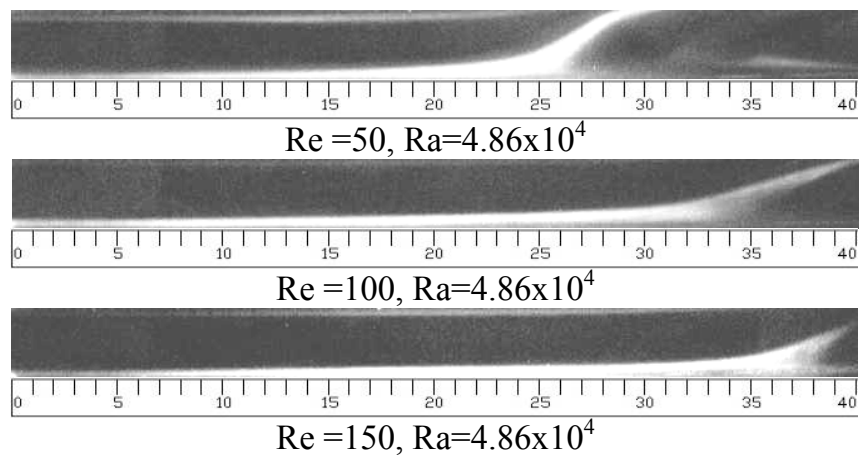

a)
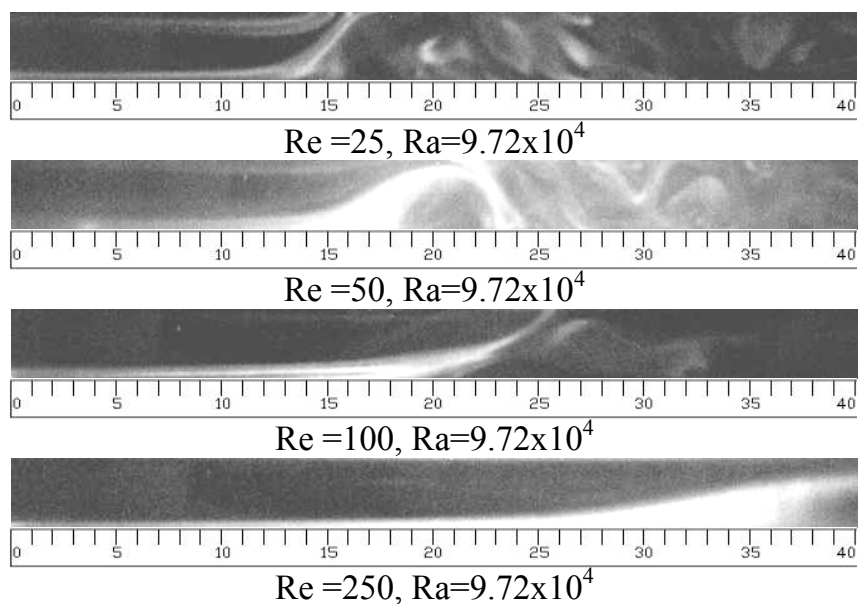

b)

Figure 4: Visualization of flow patterns at the longitudinal section $\mathrm{z}=0$ for $\mathrm{b}=20 \mathrm{~mm}$, different Reynolds numbers and: $\mathrm{Ra}=4.86 \times 10^{4}$, b) $\mathrm{Ra}=9.72 \times 10^{4}$. 


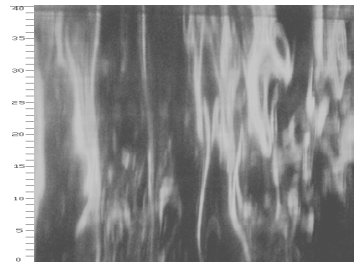

Plane $\mathrm{xz} \quad \mathrm{y}=2 \mathrm{~mm}$

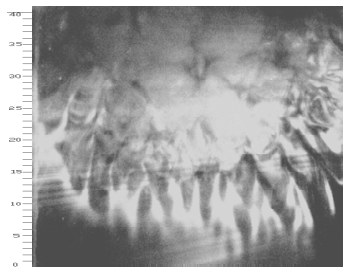

Plane $x z \quad y=2 m m$

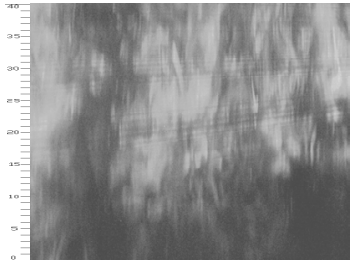

Plane $\mathrm{xz} \quad \mathrm{y}=20 \mathrm{~mm}$

a) $\mathrm{Ra}=9.20 \times 10^{5}$

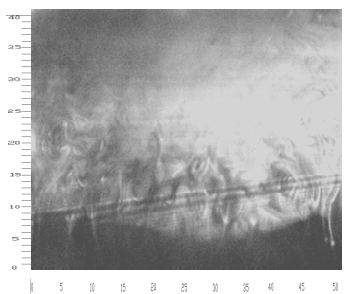

Plane $\mathrm{xz} \quad \mathrm{y}=20 \mathrm{~mm}$

b) $\mathrm{Ra}=1.85 \times 10^{6}$

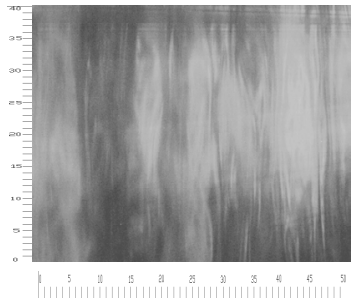

Plane $\mathrm{xz} \quad \mathrm{y}=38 \mathrm{~mm}$

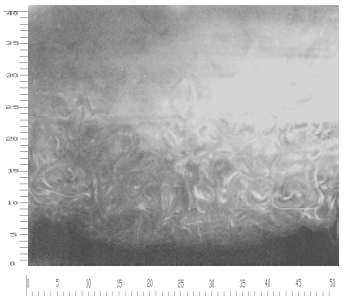

Plane $\mathrm{xz} \quad \mathrm{y}=38 \mathrm{~mm}$

Figure 5: Visualization of flow patterns in $x z$ plane for $b=40 \mathrm{~mm}, \mathrm{Re}=50$ and: a) $\mathrm{Ra}=9.20 \times 10^{5}$, b) $\mathrm{Ra}=1.85 \times 10^{6}$.

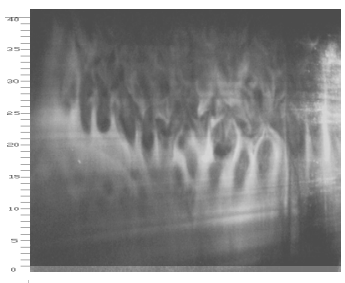

Plane $x z \quad y=2 m m$

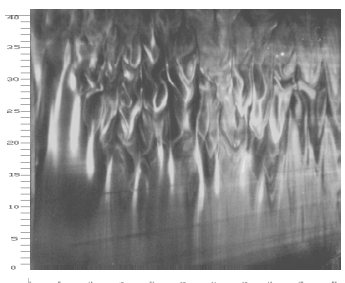

Plane $x z \quad y=2 m m$

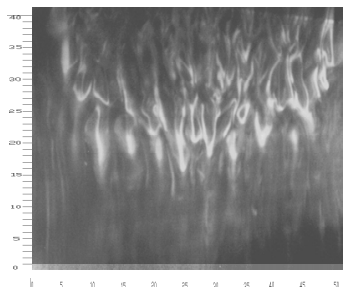

Plane $\mathrm{xz} \quad \mathrm{y}=10 \mathrm{~mm}$

a) $\mathrm{Ra}=4.86 \times 10^{4}$

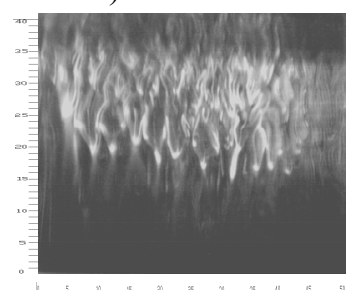

Plane $\mathrm{xz} \quad \mathrm{y}=10 \mathrm{~mm}$

b) $\mathrm{Ra}=9.72 \times 10^{4}$

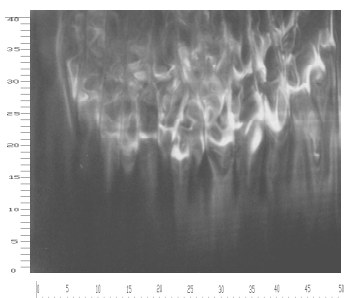

Plane $\mathrm{xz} \quad \mathrm{y}=18 \mathrm{~mm}$

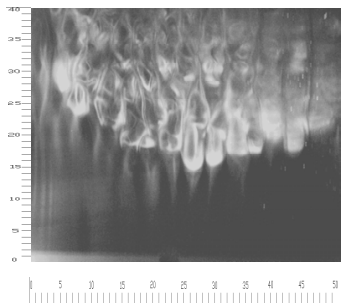

Plane $\mathrm{xz} \quad \mathrm{y}=18 \mathrm{~mm}$

Figure 6: Visualization of flow patterns in $x z$ plane for $b=40 \mathrm{~mm}, \mathrm{Re}=50$ and: a) $\mathrm{Ra}=9.20 \times 10^{5}$, b) $\mathrm{Ra}=1.85 \times 10^{6}$. 

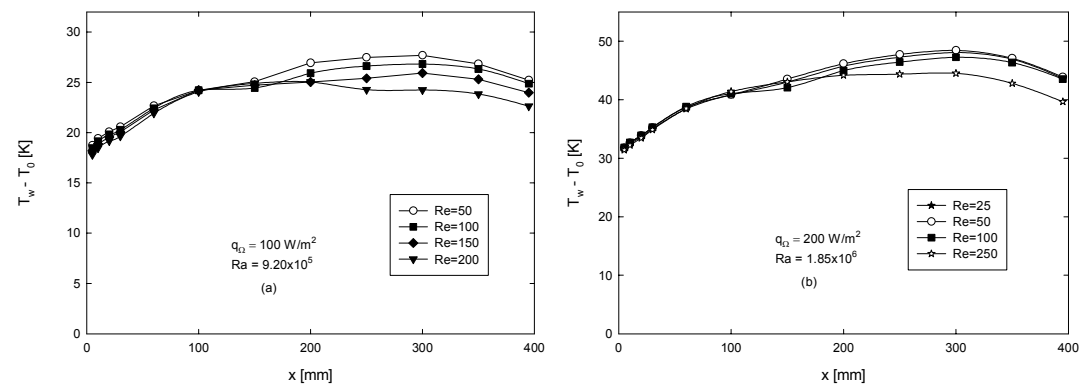

Figure 7: Wall temperature profiles for $b=40 \mathrm{~mm}$, different Reynolds numbers and: a) $\mathrm{Ra}=9.20 \times 10^{5}$, b) $\mathrm{Ra}=1.85 \times 10^{6}$.
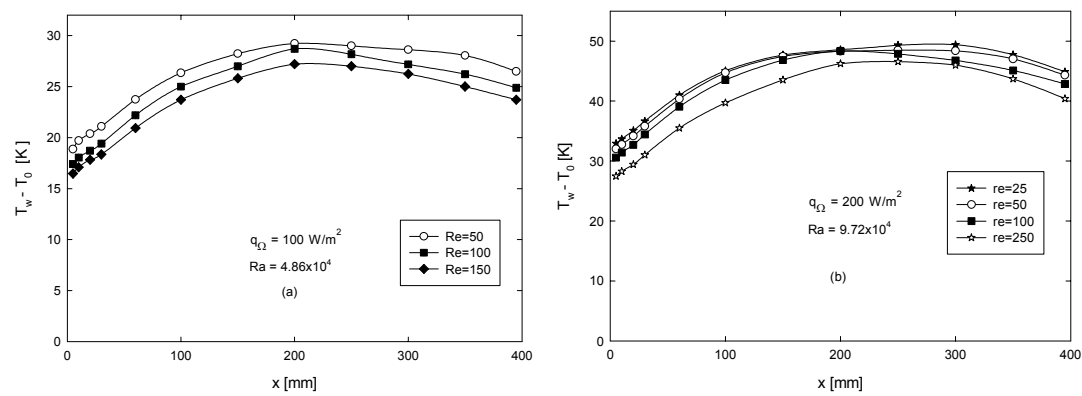

Figure 8: Wall temperature profiles for $b=20 \mathrm{~mm}$, different Reynolds numbers and: a) $\mathrm{Ra}=4.86 \times 10^{4}$, b) $\mathrm{Ra}=9.72 \times 10^{4}$.

\section{Conclusions}

An experimental investigation on mixed convection in air, in a horizontal channel, with the lower wall heated at uniform heat flux was carried out. The upper wall was unheated and heat transfer with the external ambient was allowed. Results of flow visualization highlighted the effect of secondary motions along the heated part of the channel and the separation from the lower heated plate strongly depended on the buoyancy force and forced velocity. For $\mathrm{b}=20 \mathrm{~mm}$ configuration, the separation point varies significantly and it is very close to the end of the heated channel, because inertial forces prevail on buoyancy forces as well as viscous forces prevail on inertial and buoyancy ones. Furthermore the reduction of the channel gap causes a better external heat transfer on the upper wall and low temperature values on the heated one.

\section{Acknowledgement}

This work was supported by MIUR with a 2005 PRIN grant. 


\section{Nomenclature}

\begin{tabular}{|c|c|c|}
\hline \multirow{2}{*}{$\begin{array}{l}\text { b channel spacing, } \mathrm{m} \\
\mathrm{g} \quad \begin{array}{l}\text { acceleration due to the } \\
\text { gravity, } \mathrm{ms}^{-2}\end{array}\end{array}$} & $\mathrm{x}_{\mathrm{cr}}$ & $\begin{array}{l}\text { coordinate of separation } \\
\text { point, } \mathrm{m}\end{array}$ \\
\hline & \multirow[t]{2}{*}{$\mathrm{y}$} & \multirow{2}{*}{$\begin{array}{l}\text { vertical coordinate } \\
\text { distance, } \mathrm{m}\end{array}$} \\
\hline Grashof number, Eq.(1) & & \\
\hline $\begin{array}{l}\text { heat transfer coefficient, } \\
\mathrm{W} \mathrm{m}^{-2} \mathrm{~K}^{-1}\end{array}$ & $\mathrm{z}$ & $\begin{array}{l}\text { coordinate along the width } \\
\text { of the plates, } \mathrm{m}\end{array}$ \\
\hline thermal conductivity, $\mathrm{Wm}^{-}$ & $\mathrm{W}$ & width of the plate, $m$ \\
\hline & \multicolumn{2}{|c|}{ Greek symbols } \\
\hline length of the plate, $\mathrm{m}$ & \multirow[t]{2}{*}{$\beta$} & \multirow{2}{*}{$\begin{array}{l}\text { volumetric coefficient of } \\
\text { expansion, } \mathrm{K}^{-1}\end{array}$} \\
\hline Prandtl number & & \\
\hline heat flux, $\mathrm{Wm}^{-2}$ & $v$ & kinematic viscosity, $\mathrm{m}^{2} \mathrm{~s}^{-1}$ \\
\hline Rayleigh number, $=$ Gr Pr & \multicolumn{2}{|c|}{ Subscript } \\
\hline Reynolds number, Eq.(1) & \multirow[b]{2}{*}{$c$} & \\
\hline Richardson number, Eq.(1) & & convective \\
\hline temperature, $\mathrm{K}$ & $\mathrm{k}$ & conductive \\
\hline \multirow{3}{*}{$\begin{array}{l}\text { average velocity at inlet } \\
\text { section of the channel, } \\
\mathrm{ms}^{-1}\end{array}$} & 1 & inflow \\
\hline & 0 & ambient air \\
\hline & $\mathrm{r}$ & radiative \\
\hline \multirow{2}{*}{$\begin{array}{l}\text { horizontal coordinate } \\
\text { distance, } m\end{array}$} & $\mathrm{w}$ & wall \\
\hline & $\Omega$ & Ohmic dissipation \\
\hline
\end{tabular}

\section{References}

[1] Nicolas, X., Revue bibliographique sur les écoulements de PoiseuilleRayleigh-Bénard: écoulements de convection mixte en conduites rectangulaires horizontales chauffées par le bas. International Journal of Thermal Sciences, 41, pp. 961-1016, 2002.

[2] Lin, T. F., Buoyancy driven vortex flow and thermal structures in a very low Reynolds number mixed convective gas flow through a horizontal channel. International Journal of Heat and Fluid Flow, 24, pp. 299-309, 2003.

[3] Zhang, H., Huang, X. Y., Li, H. S., Chua, L. P., Flow patterns and heat transfer enhancement in low-Reynolds-Rayleigh-number channel flow. Applied Thermal Engineering, 22, pp. 1277-1288, 2002.

[4] Bonnefoi, C., Abid, C., Medale, M., Papini, F., Poiseuille-Benard instability in a horizontal rectangular duct water flow. International Journal of Thermal Sciences, 43, pp. 791-796, 2004.

[5] Tseng, W. S., Lin, W. L., Yin, C. P., Lin, C. L., Lin, T. F., Stabilization of Buoyancy-Driven Unstable Vortex Flow in Mixed Convection of Air in a 
Rectangular Duct by Tapering Its Top Plate, ASME J. Heat Transfer, 122, pp.58-65, 2000.

[6] Ozsunar, A., Baakaya, Sivrioglu, M., Experimental investigation of mixed convection heat transfer in a horizontal and inclined rectangular channel, Heat Mass Transfer, 38, pp.271-278, 2002.

[7] Chen, S. W., Chang, C. Y., Lir, J. T., Lin, T. F., Stabilization and elimination of transient unstable mixed convective vortex flow of air in a bottom heated horizontal flat duct by top plate heating, Int. J. Heat Mass Transfer, 47, pp. 4137-4152, 2004.

[8] Liu, C. W., Gau, C., Onset of secondary flow and enhancement of heat transfer in horizontal convergent and divergent channels heated from below, Int. J. Heat Mass Transfer, 47, pp. 5427-5438, 2004.

[9] Manca, O., Nardini, S., Naso, V., Experimental investigation on mixed convection in a horizontal channel heated from below, Proc. 12th Int. Conf. Computational Methods and Experimental Measurements, pp. 705714, Malta, 20-22 Giugno 2005.

[10] Chen, S.W., Shu, D.S., Lir, J.T., Lin, T.F., Buoyancy driven vortex flow and its stability in mixed convection of air through a blocked horizontal flat duct heated from below, Int. J. Heat Mass Transfer, 49, pp.36553669, 2006.

[11] Wang, Q., Jaluria, Y, Instability and heat transfer in mixed convection flow in a horizontal duct with discrete heat sources, Numer. Heat Transfer Part A, 42, pp.445-463, 2002.

[12] Park, J. H., Chung, T. J., Yun, E. S., Kim, M. C., Choi, C. K. The onset of longitudinal vortex rolls in the thermal entrance region of plane Poiseuille flow heated with a constant heat flux, Int. J. Heat Mass Transfer, 49, pp.3708-3716, 2006.

[13] Xin, S., Nicolas X., Le Quéré, P., Stability analyses of longitudinal rolls of Poiseuille-Rayleigh-Bénard flows in air-filled channels of finite transversal extension, Numer. Heat Transfer Part A, pp.467-490, 2006.

[14] Buonomo, B., Foglia, G., Manca, O., Nardini, S., Mixed Convection in a Horizontal Channel with Heated Bottom Wall and External Heat Transfer on Upper Wall, Proc. ASME-ATI Conf., Milan 14-17 May, 2006.

[15] Moffat, R. J., Describing the Uncertainties in Experimental Results, Experimental Thermal and Fluid Science, 1, pp. 3-17, 1988. 